\title{
Y a-t-il un auteur dans la pièce ? Ethos du personnage et "figure auctoriale"
}

Is there an author in the play? The character's ethos and the "authorial" figure

\section{Jürgen Siess}

\section{OpenEdition}

1 Journals

\section{Édition électronique}

URL : http://journals.openedition.org/aad/674

DOI : 10.4000 /aad.674

ISSN : 1565-8961

Éditeur

Université de Tel-Aviv

Référence électronique

Jürgen Siess, " $Y$ a-t-il un auteur dans la pièce ? Ethos du personnage et "figure auctoriale" », Argumentation et Analyse du Discours [En ligne], 3 | 2009, mis en ligne le 15 octobre 2009, consulté le 23 septembre 2019. URL : http://journals.openedition.org/aad/674; DOI : 10.4000/aad.674

Ce document a été généré automatiquement le 23 septembre 2019.

\section{cc)}

Argumentation \& analyse du discours est mis à disposition selon les termes de la licence Creative Commons Attribution - Pas d'Utilisation Commerciale - Pas de Modification 4.0 International. 


\title{
Y a-t-il un auteur dans la pièce? Ethos du personnage et "figure auctoriale"
}

Is there an author in the play? The character's ethos and the "authorial" figure

\author{
Jürgen Siess
}

En règle générale, le public qui se rend au théâtre a certaines attentes liées au nom de l'auteur sur l'affiche. Ce nom renvoie pour lui à la personne d'un dramaturge réel, mais aussi à ce qu'il sait de lui en fonction des autres œuvres qu'il a pu voir ou lire. Si une image, même schématique, s'est au préalable formée dans son esprit, il a tendance à la rechercher dans le spectacle nouveau. Dans la communication théâtrale, la relation s'établit pourtant entre les acteurs et les spectateurs en l'absence physique du dramaturge et du metteur en scène, qui peuvent tout au plus venir saluer lorsque le rideau est tombé. Il en va de même pour le lecteur de la pièce, du point de vue duquel je me placerai ici. Il se trouve face à un dialogue, éventuellement accompagné de didascalies qui l'aident à imaginer l'espace théâtral. Dans la parole du personnage dramatique se construit une image de soi, souvent changeante et complexe, qui entre en interrelation avec celle des autres personnages de la pièce ${ }^{1}$. Cet ethos doit être clairement distingué de l'ethos 'auctorial' qu'on pourrait induire du texte dramatique dans son ensemble. Il faut donc se demander, non seulement comment une telle opération serait susceptible de se mettre en place, mais aussi dans quelle mesure elle est nécessaire pour assurer le bon fonctionnement de la communication.

\section{Réflexions méthodologiques}

\section{1. De l'analyse du récit à l'analyse du texte théâtral}

2 Rappelons que les notions de "figure de l'auteur " ou d'«auteur abstrait " ont été forgées à partir de l'instance "implied author ", auteur implicite, terme emprunté à Wayne Booth (1961) qui le définit comme le « second moi » ou la version idéale, fictive 
de l'auteur réel, mais aussi comme une figure qui se tient dans les coulisses (1983:151, 74-75). L'auteur implicite est distingué du narrateur et, dans certains cas, un net écart peut les séparer : lorsque le narrateur est doté de ses caractéristiques et de ses croyances propres, qu'on ne saurait confondre avec celles de l'auteur implicite. En proposant la notion de figure auctoriale, Maurice Couturier se démarque de Booth : il introduit une instance qui se situe " par-delà l'auteur implicite », la figure de l'auteur, «moi idéal» que l'auteur réel projette dans son texte et que, symétriquement, le lecteur façonne savamment au fil de sa lecture $(1995: 243,17)$. Cette instance auctoriale qui agence ces sens transitoires sert aussi de dénominateur commun aux auteurs implicites variant d'une œuvre à l'autre (121).

3 On peut se demander sur quels éléments les critiques se sont appuyés pour transposer sur le texte dramatique une instance empruntée au modèle narratologique ${ }^{2}$. Dans ce passage d'un plan à l'autre, les didascalies semblent avoir joué un rôle non négligeable. Une opinion encore très répandue veut en effet que dans les didascalies se dessine le profil d'un narrateur-auteur, qui renverrait à la personne réelle du dramaturge. Ainsi de nombreux critiques ont considéré les parties non-dialogales comme étant d'un autre ordre que les répliques, soutenant qu'un narrateur y apparaît sans se dissimuler et associant celui-ci à (ou le confondant avec) une instance auctoriale qui régit l'ensemble du texte. Ainsi amenés à construire dans les didascalies une double instance narrateurauteur, ils en ont induit que celle-ci peut par analogie être également décelée dans la parole des personnages (Issacharoff 1985).

4 Comme la liste des personnages et le titre, les didascalies doivent cependant être associées aux textes descriptifs, neutres. Elles semblent avoir une fonction ancillaire plutôt que directive, et servent à assister le lecteur dans son activité de compréhension-imagination. Les personnages occupant la scène/la page, les didascalies aideront le lecteur à savoir où les interlocuteurs, les « entreparleurs» (selon un terme du $16^{\mathrm{e}}$ siècle $)^{3}$, sont placés, et comment leur parole est proférée. En d'autres termes, elles appartiennent à un type de discours instable, fluctuant entre le dramatique et le descriptif. C'est dire que dans les didascalies et les autres éléments 'hors dialogue', la parole est non-subjective, elle est adressée dans un sens restreint (informatif, technique), un simple supplément nécessaire pour une intelligibilité maximale du texte, il n'y a pas d'ethos décelable ${ }^{4}$.

5 En règle générale, les modèles narratologiques dans lesquels le narrateur ou/et l'auteur implicite occupent une place de choix ne peuvent pas être transposés directement aux textes dramatiques. Ceux-ci se distinguent en ce que (1) le dialogue qui place deux ou plusieurs locuteurs en parallèle, en interrelation, en compétition, en opposition, y est omniprésent et tend à être exclusif: le personnage dramatique qui est "façonné [gestaltet] comme parlant, uniquement comme parlant» se constitue par «la disparition de la fonction narrative, par la répartition de la matière [darzustellender Stoff] sur des personnages qui se présentent et s'expriment librement " (Hamburger 1957 : 118-19, je traduis $)^{5}$; (2) il n'y a pas plusieurs niveaux de points de vue, et pas de distance - à la différence des textes narratifs où peut intervenir une instance susceptible d'agencer ces niveaux, de créer cette distance ${ }^{6}$; (3) chaque personnage a son ethos, qui se construit et se 'négocie' dans l'échange, et s'intègre à un jeu de reflets auquel le lecteur se voit confronté. Il semble donc que par les trois traits distinctifs relevés, le «drame dramatique » (Szondi) peut être conçu comme le genre de discours 
dans lequel l'effacement complet de l'auteur est constitutif'. Je comprends dans ce type de théâtre à la fois Goethe et Strindberg, Corneille et Arthur Adamov8 .

Avant de mettre à l'épreuve de deux pièces, l'une de Strindberg et l'autre de Corneille, l'hypothèse selon laquelle il n'y a pas de place pour une instance auctoriale dans le discours dramatique, il faut rappeler brièvement quelques-unes des positions les plus marquantes de la théorie du drame et de la sémiotique du théâtre sur la question de l'auteur.

\section{L'auteur dans les diverses approches du genre dramatique}

\section{1. La question de l'auteur dans la théorie du drame}

7 On peut prendre, en l'occurrence, Aristote et Platon à titre de paradigme. Dans sa Poétique, Aristote situe l'origine de la parole de la tragédie dans le poète : «les auteurs conçoivent au contraire les caractères à travers les actions » $(1990: 1449 ; 1450)$, «les [poètes] contemporains font parler [les personnages] comme des rhéteurs » (1450b). En revanche, Platon caractérise la tragédie comme "une espèce de récit» dans lequel, «retranchant les paroles du poète qui séparent les discours, on ne garde que le dialogue ", et où le poète se cache pour laisser les personnages prendre la parole à sa place (République, livre III) 9 . Ces deux positions se retrouvent par la suite tout au long de l'histoire de la poétique. Considérons le discours esthétique du $18^{\mathrm{e}}$ siècle, si riche en réflexions sur le drame. Herder (Von deutscher Art und Kunst) voit Shakespeare en génial créateur dont «le regard et le point de vue » deviennent des éléments constitutifs du monde de l'Histoire dramatisée qu'il met en scène. Quant à Schiller, lorsque (dans sa lettre à Goethe du 26-12-1796), il soutient que «face à un drame nous sommes enchaînés à la présence sensible, et sujets à une force extérieure ", le terme de force ("Gewalt ») peut se référer aussi bien au destin (à Dieu, à la passion) qu'au dramaturge lui-même. Ajoutons que, dans De la poésie épique et dramatique, Goethe et Schiller construisent l'image du " rhapsode » pour le poète épique, et celle du « mime "pour le poète dramatique. A leurs yeux, celui-ci nous plonge dans l'événement, en d'autres termes : il nous entraîne dans l'interaction dramatique.

Chez Hegel, mais aussi, plus tard et pour une autre raison, chez Brecht, l'auteur est conçu en étroite relation avec le public. Dans l'Esthétique de Hegel, la question de l'instance auctoriale est traitée plutôt implicitement, dans une dialectique qui relie entre elles les attentes du public et les capacités du dramaturge, mais aussi la norme et la "virtuosité ». Le poète, dit Hegel, semble se retirer de son œuvre, mais ce n'est qu'une apparence. S'il transparaît toujours, il gagne cependant des contours plus visibles chez les dramaturges qui cherchent à convaincre le public. Et ceux qui mettent dans la bouche des personnages leurs propres convictions (Voltaire, Lessing) sont considérés comme inférieurs aux dramaturges qui accèdent à un point de vue élevé leur permettant de voir le fond commun de tous les humains (1970: 480-81, 501-502). Situé à l'opposé de Hegel, le jeune Brecht distingue entre l'attitude " culinaire » dans le théâtre traditionnel, qu'il rejette, et celle de l'observateur actif, engagé qui considère les comportements et les rapports entre individus et groupes à l'instar de l'homme de science, dans le nouveau théâtre. Pour ce théâtre "anti-aristotélicien » il s'agit de «faire parler la réalité » $(1967: 214)$. Or, la réalité qui parle, le personnage exposé au 
regard critique du public, laissent peu de place à une instance auctoriale. D'un côté, dans le théâtre "culinaire ", on suppose que le public se laisse fasciner et cherche à s'identifier au personnage, de l'autre côté, dans le projet brechtien, on suppose que le public a un intérêt pour l'expérience scientifique qui se porte à présent non pas sur les phénomènes de la nature mais sur les rapports sociaux susceptibles d'être changés. Brecht s'intéresse moins aux images construites par le dramaturge ou le public, qu'à ce que chacun d'eux projette - sur le personnage, sa parole, son acte - et à la façon dont le personnage, sa parole, son acte, peuvent être évalués. Enfin Szondi, dans sa Théorie du drame moderne, conçoit en bon dialecticien l'auteur comme absent et présent à la fois : dans le drame traditionnel, "l'auteur est absent». Tout en étant "à l'origine du discours ", il ne parle pas : les mots « sont issus de la situation et demeurent en elle; ils ne sauraient être compris comme une parole venant de l'auteur. Le drame n'appartient à son auteur que globalement » $(1983: 14)$. Ceci implique que l'auteur cède la place aux personnages ou, plus précisément, au texte, tout en restant dans leur ombre.

9 A partir de ces exemples, on peut définir deux conceptions polarisées de la question. A l'un des deux pôles, se situe l'auteur qui s'efface - celui dont parlait Platon. A l'autre pôle, on trouve celui qui reste présent - comme, par exemple, dans la conception de Herder. Hegel, qui distingue deux types de drames, réserve cette figure au dramaturge qui prête ses convictions à tel ou tel protagoniste, tout en privilégiant celui qui s'efface au profit des personnages. Dans cette opposition, la figure dialectique de l'auteur telle que la conçoit Szondi tiendrait la place du milieu.

\section{2. La sémiotique du théâtre face à la question de l'auteur}

10 Parmi les positions défendues dans ce domaine, certaines sont proches de la conception de Szondi, même si l'orientation est à présent plutôt linguistique que philosophique. Prenons la définition d'Anne Ubersfeld :« le discours théâtral est discours sans sujet. La fonction du scripteur est d'organiser les conditions d'émission d'une parole dont il nie en même temps être responsable" $(1977: 264)^{10}$. La subjectivité est ici considérée comme problématique; la notion d'un scripteur qui organise le discours place cependant celui-ci au-dessus des personnages. Dans le théâtre (Hugo servant d'exemple), le Je du personnage reste "extérieur à l'auteur ", et le Moi de celui-ci est "caché sous les espèces non pas seulement d'un acteur mais de plusieurs acteurs " (1974 : 17). Retenons cependant la réserve importante qu'on trouve dans Lire le théâtre 3. Des personnages et du scripteur, s'interroge Ubersfeld, qui est le garant des énoncés ? La réponse reste en suspens : "peut-être le scripteur ne tranchera-t-il jamais; peutêtre - et c'est le cas le plus intéressant - laissera-t-il le litige en suspens » (1996: 54 ; je souligne). Patrice Pavis, quant à lui, considère qu'on peut induire un "sujet auctorial »des didascalies, mais aussi de la "structuration de la fable», du "montage des actions», des "perspectives et des contextes sémantiques des dialoguants ». Il précise toutefois que la résultante de ces éléments est difficile à tracer (1980 : 47). Le « sujet du discours théâtral » est éclaté en autant de sujets qu'il y a de personnages, on ne peut pas donner de contour à une figure auctoriale, qui semble se soustraire à l'analyse. Si Ubersfeld et Pavis s'interrogent sur la possible présence d'une figure auctoriale qui reste en filigrane et est difficile à reconstituer, Michael Issacharoff, en revanche, pose clairement un "archi-énonciateur", une instance qui organise et intègre les diverses énonciations et garantit leur cohérence, ou du moins leur compatibilité. Selon lui, l'auteur parle "par personnes interposées »; le locuteur, 
c'est «l'auteur, médiatisé par des comédiens imprévisibles, assistés d'un metteur en scène, qui pourra même couper le circuit » $(1985$ : 17). Il n'est que conséquent, dans cette conception, que l'énoncé didascalique signifie « c'est bien moi, auteur dramatique qui le dit...» (ibid.: 28). Ainsi, l'instance de l'archi-énonciateur apparaît comme la transposition au genre dramatique de l'«auteur implicite" tel qu'il a été élaboré à l'appui des textes narratifs, desquels Issacharoff entendait pourtant clairement distinguer les textes écrits pour le théâtre.

11 A cette dernière position s'oppose fortement celle des sémioticiens 'purs et durs' parfaitement représentés par Keir Elam. Dans l'excellent Semiotics of Theatre and Drama (1980), l'auteur - que ce soit comme figure ou comme personne réelle - est le grand absent. Le drame/spectacle est conçu comme un système autonome dans lequel il n'y a pas de place pour un garant ou un énonciateur qui puisse intervenir ou s'effacer. «The dialogic exchange [...] directly constitutes the intersubjective force of discourse » (157), « the dialogue is in the first place a mode of praxis which sets in opposition the different personal, social and ethical forces of the dramatic world "(159). Si l'on cherche, dans le système élaboré par Elam, la notion d'ethos, on la trouvera tout au plus chez les personnages. Pas la moindre trace d'un autre ego, et certainement pas d'un ethos auctorial. Ce système sémiotique offre donc un fort appui à l'hypothèse que je vais développer dans un autre cadre, celui de l'analyse du discours.

\section{3. L'analyse du discours et le texte dramatique}

Dans ce cadre précisément, la Pragmatique pour le discours littéraire (1990) semble se situer entre la position qui problématise la possibilité d'une figure auctoriale (Pavis), et celle qui l'élimine tout à fait (Elam). Dans le dispositif dramatique/théâtral, note Maingueneau, «la seule énonciation que l'on puisse valablement attribuer à l'auteur c'est l'interaction des actes de langage des personnages, une irréductible polyphonie. » Et il distingue favorablement (à l'instar de Hegel) les œuvres où l'on est confronté à tout un réseau de positions énonciatives, de celles qui entendent faire passer une thèse (1990 : 142). Il ne s'en tient pas, cependant, à cette irréductible polyphonie : il y voit au contraire un principe garantissant un sens que le public est supposé reconstruire. Aux yeux de Maingueneau, le spectateur perçoit, en plus de l'acte d'énonciation du personnage adressé à un autre personnage, l'acte d'énonciation adressé par le metteur en scène à un public spécifié et celui adressé par « l'auteur à un public virtuel » (145). Il ne franchit donc pas le pas vers la conception de l'auteur effacé, ab-strait du texte au profit des seuls personnages, de "l'interaction de leurs actes de langage » (142). Ce qui semble l'en empêcher, c'est l'archi-énonciateur d'Issacharoff, notion qu'il reprend en ces termes: "L'archiénonciateur est une instance distincte de l'écrivain, il prend en charge un réseau conflictuel de positions énonciatives » (ibid.). Dans un article de fond consacré à l'analyse du texte de théâtre, Alain Rabatel (2003) part du rapport, à la fois entre points de vue et polyphonie, et entre points de vue et double énonciation. Il maintient la notion de locuteur-dramaturge (L1), tout en précisant que c'est seulement en prenant les répliques de tous les personnages dans leur ensemble que «L1 est archiénonciateur ». Tout en ajoutant qu'il n'y a pas de garantie pour L1 d'occuper une place supérieure par rapport aux personnages et à l'interaction - la place du «surénonciateur " (2003: 13-14), qui par ailleurs peut être occupée par le personnage central (16) -, Rabatel ne met pas en question le statut même de l'archi-énonciateur. 
13 J'ai posé plus haut l'hypothèse que, dans la communication qui s'instaure entre le texte dramatique et le lecteur, il n'y a pas d'ethos auctorial. Il est temps de mettre cette hypothèse à l'épreuve des textes. Etudiant Le chemin de Damas de Strindberg et Cinna de Corneille, je relèverai la façon dont la pièce a été lue avant de réexaminer la question de l'auteur à travers une analyse du discours dramatique. Je commence par la pièce moderne, en inversant l'ordre chronologique : Damas participe (comme Torquato Tasso ou Chatterton) des pièces dont le protagoniste a été traditionnellement rapproché du dramaturge, tandis que, pour le drame classique, aucun des personnages ne peut être associé à l'auteur. Dans la mesure où le «drame de l'artiste " peut être invoqué pour soutenir la notion d'une figure auctoriale dans la pièce, il se prête à être traité en premier.

\section{Analyses}

\subsection{Le chemin de Damas}

Le premier cas de figure se caractérise par l'analogie établie entre l'ethos du personnage central et l'autoportrait de l'auteur. C'est ainsi que dans la trilogie Le chemin de Damas (1898-1901), le protagoniste a été considéré comme une projection de l'auteur réel. Il faut cependant vérifier dans le texte dans quelle mesure la pièce privilégie un héros qui renverrait à la figure de l'auteur aux dépens des autres personnages.

Dans une analyse très intéressante, Peter Szondi soutient à ce propos que Strindberg tente «de mettre au centre de l'œuvre [...] le moi propre de l'auteur [...], les personnages de la trilogie de Damas, par exemple (la Dame, le Mendiant, César) sont des émanations du moi de l'Inconnu et [...] l'œuvre est située en entier dans la subjectivité de son héros » (1983: 38 et 42). A ses yeux, la subjectivité de Strindberg est projetée sur celle du personnage central, qui à son tour se projette et se réfracte sur les autres personnages. Cette conception est redevable à Carl Dahlström (1930) qui affirme que tous les personnages de l'asile (sc. 9) sont sortis de la «mauvaise conscience de l'Inconnu » (1965: 137-38), voire que «tout naît de [son] âme même » (136) : «C'est la pièce de l'Inconnu du début à la fin, et tous les personnages émanent de son esprit comme autant de symboles des "Puissances" contre lesquelles il lutte » (ibid.: 122, je traduis). L'idée de base est, cependant, que non seulement tous les personnages sont des émanations ("Ausstrahlungen») du moi de l'Inconnu, mais aussi que le drame en tant que tel «émane tout autant du moi de Strindberg» (139). Dahlström met ainsi sur le même plan la fiction dramatique et la personne réelle avec sa psychè et les répercussions qu'elle peut avoir sur la pièce. On trouve des prolongations de cette conception chez différents metteurs en scène ${ }^{11}$ des dernières années, ainsi que dans un ouvrage récent : «Des œuvres telles que Le chemin de Damas ou Le songe sont [...] des projections [projected images] d'un dynamisme psychique » (Marker and Marker 2002 : 13, je traduis). L'affirmation que l'Inconnu renvoie en dernière instance à Strindberg s'appuie par ailleurs sur une association entre la fiction dramatique et l'autobiographie. Ainsi, lorsque Dahlström écrit que «L'Inconnu c'est Strindberg - plus tout ce qui a traversé le moi de Strindberg [the Strindberg self] », il suppose que le lecteur reconnaît la personne de l'auteur dans le personnage grâce à des images qui circulent déjà à l'époque: Strindberg le misogyne ou Strindberg le torturé. Dans ces approches, le sens 
ultime du Chemin de Damas serait à rechercher dans la vie psychique de la personne réelle du dramaturge.

Je voudrais au contraire montrer ici (1) que les ethè des personnages peuvent être placés à un même niveau et ne sont pas de pures émanations du moi de l'Inconnu ; (2) que les didascalies souvent invoquées pour faire l'hypothèse d'un "narrateur-auteur " se contentent d'offrir quelques indications nécessaires à la compréhension de l'interaction; (3) qu'il n'y a pas dans le texte une prise de position attribuable à une instance auctoriale.

Rappelons d'abord en quelques mots l'essentiel de l'action. Dès la première scène, l'Inconnu et la Dame (Ingeborg) se trouvent face à face, et ils vont réapparaître tous les deux dans la majorité des scènes de la pièce $(20 \text { sur } 34)^{12}$. Ils poursuivent la plupart du temps leur chemin ensemble, en dépit des crises que le couple traverse. Les deux partenaires se trouvent confrontés aux autres, qui leur font obstacle en tant que couple, comme le Médecin - le mari d'Ingeborg - et la Mère de celle-ci. De fortes relations d'affect et de famille lient Ingeborg et l'Inconnu à ces deux personnages et, qui plus est, dans plusieurs scènes, ceux-ci sont placés au même niveau que le couple. Le Médecin sent que sa femme cherche à le quitter, et voit qu'elle introduit son rival potentiel dans la maison (sc. 2). En même temps, il est lié à l'Inconnu par l'expiation d'un méfait commis dans le passé : quand ils étaient écoliers, l'actuel mari d'Ingeborg a été puni à la place de l'autre qui se sent coupable parce qu'il a chargé son camarade de son propre méfait (sc. 33). La Mère, quant à elle, s'immisce dans les relations qu'Ingeborg noue et dénoue, tâchant de l'éloigner de l'Inconnu et de la faire revenir à son mari (sc. 18). Ce n'est que dans une des dernières scènes de la trilogie (sc. 31) que les deux partenaires se séparent. Les scènes 28,29 et 31 montrent leur relation à son point culminant. Montant en solitaire vers le monastère où il espère trouver le salut, l'Inconnu se retrouve de nouveau face à sa compagne, ils évoquent leur passé, à présent capables de se découvrir pleinement l'un l'autre, l'un à l'autre; ils se quittent cependant, et Ingeborg encourage son compagnon à poursuivre seul son chemin. Au moment des adieux, l'Inconnu dit: «je baise la petite main que j'ai caressée et égratignée... la petite main qui m'a conduit dans le noir... m'a conduit pendant le long voyage de Damas » $(1912: 241)$.

\subsection{1}

18 Qu'en est-il, dans ce cadre, des ethè des personnages? Parmi les images de soi que chacun d'eux projette et qui jouent un rôle dans l'interaction avec l'autre, on peut distinguer celles de la Dame et du Médecin, de la Mère et du Mendiant, de l'Inconnu et de César. Sans entrer dans le détail de ces rapports complexes, on peut examiner le jeu d'images qui se crée entre l'Inconnu et la Dame dans les scènes où ils se trouvent face à face. A la fin de la première scène, par exemple, l'Inconnu se donne à voir comme oscillant entre le solitaire et l'homme cherchant la compagnie, et il reprend à son compte l'image du héros que donne de lui la Dame. Ce qu'il fait ici avec zèle n'est cependant pas exempt d'ironie :

D. [...] peut-être pourrez-vous me délivrer du loup-garou.

I. Est-ce un loup-garou?

D. C'est le nom que je lui donne.

I. Bien, je suis partant ; lutter contre des monstres, libérer des princesses, tuer des

loups-garous, c'est ça, la vie!

D. Venez, mon libérateur. 
Elle baisse sa voilette sur son visage, lui donne un baiser rapide sur la bouche et se précipite dehors. $(164)^{13}$

19 Cette première rencontre est évoquée dans la Troisième Partie (sc. 28). La Dame répond à l'Inconnu que longtemps elle ne savait pas pourquoi elle l'avait embrassé - « à présent que j'ai été loin de toi [...], à présent je vois ce dimanche après-midi où tu étais assis là comme un enfant pitoyable, abandonné » - le sentiment maternel qu'on avait toujours empêché de s'épanouir est monté en elle avec force. Aussi l'Inconnu affirme-t-il qu'elle est d'une meilleure étoffe que lui-même, et, qui plus est, tous deux vont se voir dans une parfaite réciprocité (1912: 204-210). A l'ethos de la mère qu'elle lui renvoie, l'Inconnu oppose ici les images de l'ange et de l'esprit de la terre (face positive et face négative), mais il poursuit qu'à présent, grâce à l'amour d'Ingeborg, le bien pourra vaincre le mal qu'il portait en lui, et qu'il projetait sur elle. Aussi peut-il souscrire à l'idée que sa partenaire se fait d'elle, puis, dans un jeu de reflets, se voir comme son fils et son frère à la fois. C'est dans la scène du dernier face à face (sc. 31) que l'Inconnu voit sa partenaire en médiatrice, qu'il affirme que grâce à elle il peut se réconcilier avec les femmes et avec l'humanité (237).

Les images projetées par les personnages s'intègrent dans un réseau dont aucune figure ne ressort comme prépondérante : l'image de la femme maternelle converge avec celle que projette l'Inconnu, la figure de l'homme haï par les femmes trouve son pendant dans celle du misogyne (sc. 28), et l'image de la femme sévère (la Mère) s'oppose à celle de l'homme en quête d'une partenaire maternelle (l'Inconnu). On ne peut donc pas dire que le réseau complexe d'images qui se tisse dans l'interaction privilégie l'Inconnu, ni que celles-ci soient de pures « émanations » du moi du protagoniste.

\section{1 .2}

21 Par ailleurs, on a invoqué les didascalies pour légitimer la conception d'une figure auctoriale. On peut cependant se demander dans quelle mesure les éléments 'hors dialogue' se prêtent à induire une instance clairement séparée des personnages et susceptible de régir les rapports qui se tissent entre eux. Examinons brièvement la scène d'ouverture et la scène du Banquet pour montrer quelle est la fonction des didascalies qui précèdent chacune des scènes et de celles qui ponctuent les tours de parole. Au début de "Un coin de rue » (sc. 1) on trouve une description du lieu, des bâtiments et du mobilier (pour la terrasse de café), ainsi que des éléments acoustiques, et enfin des indications minimales sur deux personnages, un homme présent dès le début et une femme qui entre en scène. Ensuite, dans les tours de paroles, sont indiqués plusieurs mouvements et gestes de la Dame et de l'Inconnu, mais aussi le baiser qu'elle lui donne à la fin et qui constitue un point culminant. Quant au «Banquet » (sc. 21), la didascalie introductive prévoit un décor festif : un petit orchestre, trois riches tables où des notables entourent l'Inconnu, en bordure une table où sont assis des gens misérables, parmi eux le Médecin et César. Quant aux indications qui sont parsemées dans les tours de parole, elles donnent des éléments qui permettent de contraster ce qui se passe et ce qui est affirmé par l'orateur et le savant fêté. A partir de l'allocution $\mathrm{du}$ "Professeur ", le fastueux décor est emporté et les convives s'éclipsent, l'Inconnu restant seul avec les misérables. Dans la première scène, on ne peut induire du texte didascalique aucune figure de "narrateur-auteur ", aucune figure de régisseur. Le cas de la scène du Banquet est plus complexe. Ne peut-on pas y déceler un régisseur qui dirige les personnages? Il faut bien voir cependant que le dialogue domine, et que la 
description du décor sert à préparer le lecteur au contraste qui, lui, est créé par les premières répliques, par la parole des personnages. Les didascalies n'ont qu'une fonction ancillaire, elles aident le lecteur à imaginer le cadre dans lequel le dialogue se déploie.

\section{1 .3}

Reste la question de savoir si, dans l'ensemble des tours de parole, on ne peut pas relever une position saillante qui permette d'induire un sens global renvoyant à un garant du sens. Pour répondre à cette question, il faut voir comment se construit le dialogue. Comme l'a bien dit Arthur Adamov, les mêmes interrogations, intimations et assertions, les mêmes mots sont repris et modulés - parfois jusqu'à satiété - par la Dame ou la Mère, l'Inconnu ou le Médecin (1982: 85). Prenons les scènes où ces deux personnages masculins se trouvent face à face. Lors de leur première rencontre (sc. 2) le Médecin nie connaître l'Inconnu ; plus tard il le menace (sc. 16); enfin il l'accuse de l'avoir «assassiné » deux fois (sc. 24). Dans ces trois occurrences, ainsi que dans la scène du Banquet (sc. 21), le thème raison-folie est repris dans l'échange entre le Médecin et son rival. Ce sont ces reprises et variations qui sous-tendent le dialogue du Chemin de Damas (Adamov parle de «brassage involontaire » des mêmes thèmes - 1982 : 103), et non des raisonnements susceptibles d'amener une solution. Aussi a-t-on à faire à des affects qui traversent la parole des personnages plutôt qu'à des arguments.

Qu'en est-il d'une possible prise de position propre à l'Inconnu qui dominerait celles des autres personnages? Sa prépondérance est compromise par les nombreuses critiques émises à l'égard du protagoniste : la Mère lui adresse de graves reproches d'être irresponsable, et animé par l'« esprit infernal de la révolte » (sc. 10), le Médecin l'accuse et le menace (sc. 18), César le traite de charlatan (sc. 21), et sa Fille le laisse interdit, quand elle lui fait entendre sans ambages que toute sa vie est un échec (sc. 28). Si l'Inconnu évoque ses expériences douloureuses, exprimant ses doutes sur ce qu'il a vécu et ses angoisses sur ce qui l'attend, le Médecin, la Mère et la Dame en font autant. Aussi ne saurait-on distinguer chez l'Inconnu une position supérieure qui focaliserait toute l'attention en lui subordonnant le discours des autres personnages.

On peut, bien sûr, se demander si le dénouement - la conversion de l'Inconnu -, qui peut être considéré aussi bien comme un point culminant que comme une chute, marque une prise de position qui indiquerait malgré tout une présence auctoriale. Celle-ci fonctionnerait alors comme le garant du sens. L'analogie établie entre la personne de Strindberg et l'Inconnu n'apporte cependant pas la solution à ce que la pièce laisse en suspens. Le texte est plutôt fait pour engager le lecteur à prendre position : il est invité à réfléchir au choix final de l'Inconnu, mais aussi à la scène des adieux, qui est une fausse fin. Une conclusion - parmi d'autres - pourrait être que les scènes de la conversion, qui succèdent à celle des adieux, constituent une dérive, que le texte tend ici à une réconciliation qui semble contraire à la dynamique par laquelle il s'est jusquelà distingué. Mais on peut aussi retenir l'indécision marquante du texte jusque dans les dernières scènes. La conversion qui y est mise en avant garantit-elle une résolution des conflits, ou l'entrée au monastère n'est-elle qu'une fuite? Avec ces scènes de conversion, le texte laisse le lecteur face au choix - de les lire comme fournissant à la pièce son sens ultime, ou de laisser « le litige en suspens ». 
analyse tend à montrer qu'aucun ethos auctorial ne peut être induit de ce texte dramatique, qu'il s'agisse d'une projection de l'auteur réel ou de la présence à travers le dialogue et les didascalies d'un garant du sens.

\subsection{Cinna ou la clémence d'Auguste (1641)}

On parvient ainsi au théâtre où aucun des protagonistes ne peut être identifié avec le dramaturge. Ce cas est exemplifié dans les drames classiques de Corneille, de Racine ou de Schiller, et en particulier dans Cinna ou la clémence d'Auguste de Corneille, que j'examinerai ici de plus près. La pièce se prête sans nul doute à être contrastée avec le Chemin de Damas. Lorsque, dans les études strindbergiennes, on établit une relation entre le protagoniste de la pièce et le dramaturge, c'est l'image de l'auteur telle qu'elle s'est formée en-dehors du texte qui semble cautionner l'analogie. Dans les études cornéliennes, au contraire, on soutient en général qu'un fossé sépare le dramaturge de ses personnages: "le fossé entre l'homme et l'œuvre est tel qu'il rend tout rapprochement, toute projection de ce genre, extrêmement problématiques " (Defaux 2004 : 721). On a donc le plus souvent exclu tout rapport entre le bourgeois provincial et sans éclat, et le sublime héros cornélien. Dans cette perspective, une partie de la critique (comme Doubrovsky 1963, par exemple) a également refusé d'établir un lien quelconque entre la vision chrétienne du monde qui caractériserait Corneille, et l'exigence éthique qui se traduirait dans les tragédies. Defaux, après bien d'autres, considère au contraire qu'il faut faire un rapprochement entre la position de l'auteur telle qu'elle résulte de sa formation chez les jésuites, et le sens global de sa pièce. En d'autres termes, il suggère de remonter à la personne réelle de l'auteur pour interpréter le texte à bon escient.

Fumaroli, dans sa grande étude sur "rhétorique et dramaturgie cornéliennes », (1996), se concentre sur les personnages-orateurs. Il voit cependant une projection du dramaturge dans un de ses personnage lorsqu'il écrit : « Il y a beaucoup de Don Sanche dans Corneille, [...] son théâtre est donc déjà, et au sens propre, autobiographique, découverte et création de soi-même et de sa propre vie par une œuvre littéraire » (21). Il en va de même lorsqu'il dit : «Par la bouche de ses personnages, mieux que les hauts magistrats orateurs du parlement, il harangue la cour et le peuple, traitant sous le rôle de la fiction les plus hautes questions de la morale politique » (26). Fumaroli réintroduit ici de biais l'idée du personnage porte-parole du dramaturge qui ne semble guère compatible avec l'idée qu'un fossé infranchissable sépare l'homme et l'œuvre. L'approche selon laquelle il faut remonter à l'éducation de l'écrivain et aux positions d'un garant du sens retrouve ainsi ses droits lors même qu'aucun amalgame ne peut être fait entre le dramaturge et ses protagonistes.

\subsection{1.}

Commençons l'analyse en examinant les didascalies. Elles sont minimes dans Cinna, où le dialogue est omniprésent : une indication de lieu au début (Rome), et cinq indications de geste ou de mouvement parsemées dans les tours de parole. ${ }^{14}$ "Rome» est tautologique pour le lecteur de l'époque, dès lors qu'il a pris connaissance du nom d'Auguste (dans le titre et la liste des "Acteurs »), et il devra induire le lieu précis, le palais de l'empereur, du dialogue qui suit. Quant à la liste des personnages, elle comporte surtout des caractéristiques politiques - empereur, impératrice, (autre) chef

Argumentation et Analyse du Discours, 3 | 2009 
de la conjuration, proscrit - ; les caractéristiques familiales - fils, fille - sont en nombre inférieur. Tous ces éléments sont exposés de la façon la plus neutre, ils indiquent le minimum dont le lecteur a besoin pour pouvoir situer le dialogue : on ne trouve aucune trace d'un « narrateur-auteur », d'un régisseur.

Le minimalisme des éléments 'hors dialogue' permet donc de réserver toute l'attention au dialogue seul. On dira que, dans Cinna, toute l'action ${ }^{15}$ est dans ce discours, dont l'image de soi des personnages constitue un élément intégrant. Mais tandis que chez Strindberg, on peut observer des images, similaires ou opposées, qui circulent d'un personnage à l'autre, chez Corneille l'ethos des personnages est directement lié à l'argumentation dans laquelle chacun d'entre eux s'engage. Dans Le chemin de Damas, les personnages tentent à la fois de nouer une relation à l'autre et de se constituer une identité : ce désir sous-tend la dynamique du jeu de reflets des ethè dans une pièce qu'on a souvent envisagée en termes de psychologie. Dans Cinna, au contraire, l'image de soi participe d'une entreprise de persuasion au sein des divergences ou des oppositions qui se font entendre. Cinna et Auguste, Emilie et Maxime ont chacun une position qu'ils entreprennent de défendre dans une confrontation avec l'autre, ou qu'ils mettent en question dans une délibération intérieure. Le maniement de l'ethos participe donc bien de l'art rhétorique cornélien si souvent mis en valeur.

\subsection{2.}

30 L'argumentation, qui occupe la plus grande partie du dialogue, prend souvent la forme d'une joute oratoire. Pour les conspirateurs, il s'agit de légitimer leur but à l'encontre d'Auguste, et c'est dans ce cadre que les ethè remplissent une fonction primordiale. Ainsi, Cinna justifie le projet de tuer le tyran par la gloire attachée à l'acte héroïque, mais aussi par le désir d'instaurer la république considérée comme un régime supérieur à l'empire. Il se présente en homme courageux prêt au sacrifice. Emilie, quant à elle, se présente en fille fidèle à la mémoire de son père tué sur l'ordre d'Octave-Auguste. Confrontée à l'hésitation de Cinna au moment où Auguste se montre généreux à son égard, Emilie, qui persiste dans leur entreprise, accepte l'image de la femme perfide que lui renvoie Cinna (III, 4) pour la retourner à son profit: «La perfidie est noble envers la tyrannie » (v. 974). Au seul intérêt particulier, Emilie oppose la dignité d'être libre, but majeur qui justifie le régicide que son amant taxe à présent de perfidie. Emilie : «Je me fais des vertus dignes d'une romaine.» Cinna : «Un cœur vraiment romain... » Emilie : «[...] fuit plus que la mort la honte d'être esclave» (v. 978-81). En invoquant la liberté républicaine à l'encontre de Cinna près de se réconcilier avec Auguste (v. 1000-1002), Emilie se place sur le plan de l'intérêt général ; combattre pour la république est un objectif qui rehausse celui de venger la mort du père. Une série de retournements caractérisent le dialogue. Cinna se considère comme traître à l'égard d'Auguste, tandis qu'Emilie lui reproche au contraire de trahir son amante (v. 931 et ss.) ; lorsqu'il se dit ingrat par rapport à un souverain généreux, Emilie lui oppose l'image du régicide généreux parce qu'il apporte la liberté à tout un peuple (v. 969 et ss.). Dans cette joute oratoire, l'argumentation s'appuie sur la double face des images, qui sont soumises à une série d'inversions pour servir le but poursuivi par Emilie.

31 La jeune femme joue également des renversements d'images avec Auguste lorsque, en plaidant sa cause, elle inverse le rapport accusée/accusateur. Sommée de s'expliquer devant Auguste qui la traite de parricide, elle réplique qu'elle n'a fait que suivre son exemple $(\mathrm{V}, 2)$. Elle se présente cependant comme supérieure à lui puisque le mobile de 
son adversaire, l'ambition, est moins noble que le sien, le « juste courroux » à l'égard de l'assassin (v. 1602-1603). Elle se pose de plus en bienfaitrice : l'acte de venger la mort de son père doit permettre de réinstaurer la république. En faisant entendre que c'est à elle que le titre de bienfaiteur revient légitimement, elle réclame ainsi pour elle-même l'image valorisante qu'Auguste s'est attribuée. Dans l'ensemble, elle travaille à renverser la position de départ des deux interlocuteurs. C'est désormais à Auguste qu'il revient de se défendre face aux accusations de celle qu'il avait chargée du crime de parricide.

Après l'intervention de Livie en faveur de son époux, Emilie opère un revirement de situation en passant du réquisitoire à un aveu de culpabilité où elle se présente en principale responsable du projet meurtrier et en dangereuse séductrice : «Si j'ai séduit Cinna, j'en séduirai bien d'autres » (1622). Cet ethos de séductrice sur laquelle retombe toute la culpabilité du fatal dessein s'avère cependant être le levier d'une nouvelle joute oratoire entre les deux amants au cours de laquelle chacun réclame pour lui l'héroïsme glorieux. Par un retournement d'images effectué dans les discours adressés à Auguste, Cinna s'approprie le titre de séducteur dont se parait Emilie: il aurait tenté de la séduire (v. 1625) par une offre de vengeance : « Elle n'a conspiré que par mon artifice,/ J'en suis le seul auteur, elle n'est que complice » (v. 1637-8). Ainsi, en disculpant l'autre, chacun revendique pour lui le statut d'initiateur du régicide et la gloire qui s'y attache. Ce qui apparaît comme une rivalité déclarée doit cependant être entendu comme une tentative de sauver l'autre, ce qui ajoute encore au caractère glorieux de l'acte. Cette double revendication est résolue par Emilie dans la construction finale d'une image de couple uni, un Nous qui s'oppose au Jede l'empereur et qui marque la convergence parfaite des buts poursuivis par les deux amants (v. 1651-54) :

De nos parents perdus le vif ressentiment

Nous apprit nos devoirs en un même moment.

En ce noble dessein nos cœurs se rencontrèrent,

Nos esprits généreux ensemble le formèrent, [...].

La fille du tuteur assassiné revendique à travers ce Nous une place égale à celle de l'empereur par la projection d'une image de noblesse et de générosité ${ }^{16}$ qui l'emporte sur celle dont se pare Auguste lorsqu'il lui rappelle les bienfaits qu'elle lui doit.

L'image de soi n'intervient pas seulement dans les joutes oratoires qui se déroulent dans l'argumentation "juridique ", avec ses plaidoyers et ses réquisitoires. Elle joue aussi un rôle dans la délibération intérieure du personnage. Pris dans un conflit entre deux solutions possibles, celui-ci pèse sa décision en se confrontant à l'image de sa personne que l'autre lui renvoie, et qui met en doute l'idée qu'il avait - et qu'il entend donner - de lui-même. Ainsi, Cinna, après avoir été confronté à Auguste contre lequel il conspire, se dit tiraillé entre deux partis qui s'excluent mutuellement et commence à douter de son entreprise : «En ces extrémités quel conseil dois-je prendre ?/ De quel côté pencher, à quel parti me rendre ?» (v. 873-74). Dans son monologue, il est déchiré entre sa crainte de se montrer ingrat à l'égard du maître magnanime, et son désir d'apparaitre en libérateur glorieux. Il lui est également difficile de rester fidèle à l'engagement contracté auprès de l'amante (III, 3) dès lors qu'il se voit lui-même en traître: «O coup, ô trahison trop indigne d'un homme!» (v. 885). Le personnage adresse à soi-même arguments et contre-arguments, et les facettes de l'ethos sont des éléments constitutifs de cette délibération auto-adressée. 

concernant la décision qu'il doit prendre suite à la découverte de la conspiration menée contre lui. Il pèse le pour et le contre d'un assouplissement du régime et d'une forme de partage du pouvoir. Plusieurs facettes de son image sont invoquées par le souverain. A la première image de la victime pitoyable parce que détestée par ses propres amis, Auguste en vient à opposer celle du dirigeant sanguinaire (v. 1132-1138) et du bourreau qui, en violant les droits, a lui-même suscité la conspiration (v. 1142-1144). Il se présente en ingrat qui doit payer ses fautes en renonçant au pouvoir. Cette autoaccusation se transforme néanmoins en réquisitoire qui renverse les conclusions auxquelles il était parvenu à travers la condamnation morale de sa propre personne. $\mathrm{Au}$ lieu de se présenter en coupable qui doit payer ses méfaits, il se retourne contre Cinna qui le «traite en criminel » et dont la trahison «fait seule [son] crime» (v. 1153). Auguste s'érige alors en juge suprême qui doit punir celui qui conspire «contre le bonheur de l'Etat» (v. 1156). Un revirement supplémentaire transforme cependant l'image du juge-exécuteur en celle de l'homme lassé de faire couler le sang et déterminé à se donner une mort héroïque - pour revenir sur l'option de la vengeance et sur l'image du bourreau. Entre ces facettes de l'image qu'il projette de lui-même, Auguste ne parvient pas à trancher et se présente en être déchiré : « $O$ rigoureux combat d'un cœur irrésolu/ Qui fuit en même temps tout ce qu'il se propose !» (v. 1188-89). Les ethè jouent ainsi un rôle déterminant aussi bien dans la confrontation des personnages qui défendent des positions opposées, que dans la délibération intérieure où le personnage s'adresse à lui-même.

qui marque dialogues et monologues tout au long de la pièce débouche sur une solution au sein de laquelle se dissolvent les oppositions et les contradictions qui sous-tendent la délibération intérieure ou la joute oratoire. La scène du dénouement $(\mathrm{V}, 3)$ enchaîne les images du maître de soi-même, de l'ami magnanime et du père, toutes au profit d'Auguste : «Soyons amis, Cinna [...]» - « Aime, ma fille, Cinna [...]/ Te rendant un époux, je te rends plus qu'un père » (v. 1701, v. 1711-14). L'apogée est marquée par l'image du père qui, en dépit de l'offense, fait le bonheur de sa fille - père en ami des deux enfants qu'il adopte en les mariant, ou, si l'on suit la suggestion de Fumaroli, père qui est adopté par le couple... C'est la convergence harmonieuse des images d'Auguste à ses yeux comme aux yeux des autres, qui est le signe de la résolution finale des confrontations verbales comme des déchirements intérieurs.

Ainsi, les caractéristiques relationnelles et politiques se superposent et, qui plus est, les relations politiques (souverain-favoris) sont imaginées comme des relations familiales (père-enfants). Celles-ci semblent rehausser les rapports de pouvoir, et leur conférer une légitimité quasi-naturelle. Auguste se réserve le lien du père aux enfants, relation aussi forte qu'exclusive. On retrouve donc la haute importance qui revient aux images de soi et de l'autre dans le développement et la résolution du texte dramatique ${ }^{17}$.

Dans l'ensemble, l'argumentation des personnages porte en elle des questions d'éthique qui vont bien au-delà des positions défendues par chacun d'entre eux. On peut en effet considérer que le texte suscite une interrogation. Les arguments qui s'opposent les uns aux autres invitent à se demander comment distinguer l'action légitime de l'action illégitime, et comment décider des valeurs qui guident et cautionnent les choix de comportement. A la position qui voit dans la pièce une interrogation ouverte s'oppose la lecture qui voit une solution dans l'acte de clémence final d'Auguste (dans son interprétation laïque ou chrétienne), ou encore dans la synthèse entre l'empire et la 
république réalisée par le dénouement (Merlin 1994 : 303). L'essentiel est ici que, en dépit de la différence des points de vue sur la pièce, il ne semble pas possible d'avoir recours à un ethos auctorial, en l'occurrence un "archi-énonciateur " qui renverrait à l'auteur. Encore moins que dans Le chemin de Damas, on peut, dans Cinna, induire une instance auctoriale: les personnages (ou « héros-orateurs») semblent parler en leur nom propre, et c'est à leur parole seule que le lecteur est confronté ${ }^{18}$. L'instance auctoriale disparaît derrière un discours qui n'est attribuable qu'aux seuls personnages, comme si leur dialogue incitait le public à prendre position par rapport aux divers arguments et contre-arguments. Dans cette interaction entre le texte et le public, il n'y a pas de place pour une figure d'auteur.

\section{En guise de conclusion}

On a pu voir que cette exploration d'une figure d'auteur dans le discours dramatique se fonde sur les textes seuls, en faisant abstraction de la représentation théâtrale. La communication dramatique s'établit dans et par l'interrelation des personnages et du lecteur, alors que la réalisation scénique fait intervenir une multiplicité d'agents: metteur en scène, auteur, acteurs, conseiller littéraire, régisseur, décorateur, pour ne parler que du pôle de la production. Dans cette optique, on a l'impression que l'auteur est un des éléments de la production théâtrale conçue comme un tout : il s'intègre dans un groupe étroitement soudé dont il est un des agents principaux. Contrairement à la production théâtrale, le texte dramatique, activé par le lecteur seul, ne confronte celuici qu'aux personnages et à leur dialogue. Le lecteur est amené à reconstruire l'image de soi qu'ils projettent et à suivre le jeu des ethè qui sous-tend leur interaction. Dès lors, il n'y a pas lieu de chercher une figure auctoriale dissimulée derrière le dialogue. Cela ne signifie pas, bien sûr, que l'on ignore la personne réelle de l'auteur ou l'écrivain dans le champ, ni que l'on nie l'existence d'une image d'auteur fabriquée par l'institution.

\section{BIBLIOGRAPHIE}

Adamov, Arthur. 1982 [1955]. Strindberg (Paris : L'Arche)

Amossy, Ruth (éd.). 1999. Images de soi dans le discours (Lausanne : Delachaux et Niestlé)

Aristote. 1990. Poétique (éd. M. Magnien) (Paris : L. G. F.-Le Livre de Poche)

Barthes, Roland. 1973. Le plaisir du texte (Paris : Seuil)

Booth, Wayne C. 1983 [1961]. The Rhetoric of Fiction (Harmondsworth : Penguin)

Brecht, Bertolt. 1967. Gesammelte Werke 15 (Frankfurt/M. : Suhrkamp)

Corneille, Pierre. 1963. Cuuvres complètes (éd. A. Stegmann) (Paris : Seuil)

Couturier, Maurice. 1995. La figure de l'auteur (Paris : Seuil)

Dahlströhm, Carl E. W. L. 1965 [1930]. Strindberg's Dramatic Expressionism (New York : Blom) 
Defaux, Gérard. 2004. « Cinna, tragédie chrétienne ? Essai de mise au point », MLN 119, 718-765

Despierres, Claire, Hervé Bismuth, Mustapha Krazem \& Cécile Narjoux (dir.). 2009. La Lettre et la scène. Linguistique du texte de théâtre (Dijon : Éditions universitaires de Dijon)

Elam, Keir. 1980. The Semiotics of Theatre and Drama (London : Methuen)

Doubrovsky, Serge. 1963. Corneille ou la dialectique du héros (Paris : Gallimard)

Forestier, Georges. 1996. Essai de génétique théâtrale. Corneille à l'œuvre (Paris : Klincksieck)

Fumaroli, Marc. 1996 [1990]. Héros et orateurs. Rhétorique et dramaturgie cornéliennes (Genève : Droz)

Hamburger, Käthe. 1957. Die Logik der Dichtung (Stuttgart : Klett-Cotta)

Hegel, Georg F. W. 1970. Werke 15 : Ästhetik (Frankfurt/M. : Suhrkamp)

Issacharoff, Michael. 1985. Le spectacle du discours (Paris : Corti)

Kerbrat-Orecchioni, Catherine. 1980. L'énonciation : de la subjectivité dans le langage (Paris : Colin)

Kindt, Thomas \& Hans-Harald Müller. 2006. The Implied Author. Concept and Controversy (Berlin : de Gruyter)

Leiner, Wolfgang \& Bayne, Sheila. 1984. «Cinna ou l'agenouillement d'Emilie devant la clémence d'Auguste », Leiner, W. (ed.). Onze études sur l'image de la femme dans la littérature française du $17^{e}$ siècle (Tübingen : Narr), 146-163

Lochert, Véronique. 2004. « Entreparleurs, acteurs, personnages; who's who dans les listes de dramatis personae (XVIe XVIIe siècles) », communication U. Paris 7 (19-11-2004)

Maingueneau, Dominique. 1990. Pragmatique pour le discours littéraire (Paris : Bordas)

Marker, Frederick J. \& Marker, Lise-Loue. 2002. Strindberg and Modernist Theatre. Post-Inferno Drama on the Stage (Cambrigde : Cambrigde University Press)

Merlin, Hélène. 1994. Public et littérature en France au 17e siècle (Paris : Les Belles Lettres)

Patron, Sylvie. 2009. Le narrateur (Paris : Colin)

Pavel, Thomas. 1976. La syntaxe narrative des tragédies de Corneille (Paris : Klincksieck)

Pavis, Patrice. 1980. Dictionnaire du théâtre. Termes et concepts de l'analyse théâtrale (Paris : Editions sociales)

Rabatel, Alain. 2003. «Le problème du point de vue dans le texte de théâtre ", Pratiques 119/120, 7-33

Strindberg, August. 1991. Till Damaskus (ed. G. Ollen) (Stockholm : Norstedts)

Strindberg, August. 1997. Théâtre complet 5 (Paris : L’Arche)

Strindberg, August. 1912. Nach Damaskus (München \& Leipzig : Müller)

Szondi, Peter. 1983 [1963]. Théorie du drame moderne 1880-1950, trad. P. Pavis (Paris : L’Age

d'Homme)

Ubersfeld, Anne. 1974. Le roi et le bouffon. Etude sur le théâtre de Hugo de 1830 à 1839 (Paris : Corti)

Ubersfeld, Anne. 1977. Lire le théâtre (Paris : Editions sociales)

Ubersfeld, Anne. 1996. Lire le théâtre 3 : Le dialogue de théâtre (Paris : Belin) 


\section{NOTES}

1. Voir Amossy 1999, ouvrage de référence sur l'ethos.

2. Ce modèle est critiqué par Thomas Kindt et Hans-Harald Müller (2006).

3. «Entreparleurs, acteurs, personnages; who's who dans les listes de dramatis personae (XVIe XVIIe siècles) », communication de Véronique Lochert, U. Paris 7, 19-11-2004

4. Krazem (Despierres et al. $2009: 130,131$ ) soutient - comme moi - que la figure de l'auteur n'est pas inscrite «directement dans les structures linguistiques » du discours didascalique, ceci ne l'empêche cependant pas d'affirmer peu après - ce qui me semble paradoxal - que l'omniscience du commentateur est un fait incontournable et que l'auteur «ne cache pas qu'il connaît le processus à venir ".

5. Je traduis. - Déjà pour Aristote la tragédie « est une imitation faite par des personnages en action et non par le moyen d'une narration » (Poétique 1450 a).

6. Par contre, Rabatel (2003) propose de distinguer, à l'intérieur même des relations entre personnages, des sur- et sous-énonciateurs (p. ex. lorsqu'un personnage rapporte la parole d'un autre personnage, il serait en position de sur-énonciateur).

7. Szondi oppose à ce type de drame le drame « épique » (Brecht et Thornton Wilder) dans lequel une instance narrative, le "moi épique», est introduite, ce qui est le signe majeur d'un changement profond. Le "théâtre épique » du $20^{\mathrm{e}}$ siècle nécessiterait un développement à part que je ne peux donner ici.

8. Acte sans paroles de Beckett constitue un cas limite: on a pu le qualifier tantôt de pantomime, tantôt de texte de théâtre exclusivement didascalique. Il faudrait reposer la question de savoir dans quelle mesure certains dispositifs dramatiques donnent un autre statut aux didascalies. Je pense aux pièces de Bernard Koltès analysées par André Petitjean, au colloque «Style, langue et société » (Cerisy, 10-17 sept. 2009).

9. Cité d'après Aristote (1990: 138)

10. Pour la notion de sujet voir Kerbrat-Orecchioni $1980: 179-80$.

11. Jobst Langhaus, Scala Basel/Werkbühne Berlin, 23-3-2001; Ivan Dobchev, Theatrelaboratory, Sofia 11-10-2007 ; Sabine Harbeke, Theater Kiel, 1-10-2008. Une notable exception : Max Lundqvist (Teaterkaos Stockholm, 2004) qui fait jouer la pièce dans un dispositif de manège de cirque.

12. La répartition en 34 scènes (I : 1 à $17, \mathrm{II}: 18$ à 26 , III : 27 à 34 ) suit celle de la traduction Schering autorisée par Strindberg (1912).

13. J'ai ponctuellement changé la traduction du Théâtre complet (1997) à partir de la version allemande (1912) et du texte original (Strindberg 1991).

14. II, 1 Tous se retirent, à la réserve de Cinna et de Maxime; IV, 1 Il lui parle à l'oreille et Polyclète rentre ; IV, 3 Elle est seule; V, 3 A Maxime

15. Cinna, descendant de Pompée qui a été tué sur l'ordre d'Octave - à présent Auguste empereur - conspire contre celui-ci, engagement qu'il a contracté en même temps auprès de son amante, Emilie qui tâche de venger la mort de son père. La conspiration est découverte, Auguste infléchit Cinna à renoncer à son projet et se montre clément à l'égard du jeune couple.

16. Voir Forestier (1996: 228) et Leiner \& Bayne (1984: 149).

17. Pour Forestier toute la pièce repose sur un va-et-vient entre deux images du prince : l'image tyrannique du passé qui ressurgit, et l'image royale qu'Auguste établit dès l'acte II (1996:227).

18. Ou, pour reprendre la notion de garant du sens : il y a autant de garants du sens que de personnages, et la plurivocité ne peut être ramené à une voix d'auteur. 


\section{RÉSUMÉS}

Cet article entend démontrer que dans le genre dramatique, aucune instance auctoriale n'intervient dans la communication qui s'établit entre le texte et le lecteur. Dans les textes dramatiques, le dialogue est omniprésent, la fonction narrative disparaît, chaque personnage construit son ethos qui est 'négocié' dans l'échange, et aucun ethos auctorial n'est décelable dans les didascalies. Un rappel des positions les plus marquantes de la théorie du drame et de la sémiotique du théâtre sur la question de l'auteur sont suivies d'un bref examen du Chemin de Damas de Strindberg et du Cinna de Corneille. Dans une pièce où le protagoniste a été perçu comme une projection de la personne réelle de l'auteur, et dans une tragédie où un tel rapprochement est impossible, on examine le jeu des ethè pour voir dans quelle mesure ils permettent d'induire une figure auctoriale ou un garant du sens.

The objective of this article is to demonstrate that in the dramatic genre, the authorial agent does not play any role in the text/reader communication. In the dramatic texts, dialogue is omnipresent, the narrative function disappears, each character builds an ethos 'negotiated' in the exchange, and no authorial ethos is to be found in the stage directions. A reminder of various stances on the question of the author as elaborated in the most striking theories of drama and in the semiotics of theatre is followed by a short analysis of To Damascus by Strindberg and of Corneille's Cinna. The interplay of ethè is thus examined in a play where the protagonist has been perceived as a projection of the real author, and in a tragedy where this connection is impossible, in order to check whether they allow for any authorial figure or any ultimate warrant of the text's meaning.

\section{INDEX}

Mots-clés : dialogue, didascalie, dramaturge, ethos, personnage, sémiotique, théorie du drame Keywords : character, dialogue, dramatist, ethos, semiotics, stage direction, theory of drama

\section{AUTEUR}

\section{JÜRGEN SIESS}

Université de Caen, ADARR 